\title{
Lightweight Body in White Design Using Topology-, Shape- and Size Optimisation
}

\author{
Christophe Bastien ${ }^{1}$, Jesper Christensen ${ }^{2}$, Mike V Blundell ${ }^{3}$, Jakovs Kurakins ${ }^{4}$ \\ ${ }^{1}$ Coventry University, christophe.bastien@ coventry.ac.uk, Priory Street, Coventry, CV1 5FB, UK \\ 2 jesper.christensen@coventry.ac.uk \\ ${ }^{3}$ mike.blundell@coventry.ac.uk \\ 3.kurakins@hotmail.de
}

\begin{abstract}
As focus on the world climate rises, so does the demand for ever more environmentally friendly technologies. The response from the automotive industry includes vehicles whose primary propulsion systems are not based upon fossil fuels. On this basis a Low Carbon Vehicle Technology Project (LCVTP), partly funded by the European Regional Development Fund (ERDF), has been completed. The project included designing a lightweight Body In White (BIW), specifically tailored to suit the drive train and general packaging requirements associated with a Hybrid Electric Vehicle (HEV). The future opportunities for optimising the new lightweight vehicle architecture have been investigated using a technique entitled topology optimisation, which extracts the idealised load paths for a given set of load cases, followed by a shape- and size optimisation in order to provide local areas of the vehicle with more definition.

An appropriate shape- and size optimisation process for frontal crashworthiness scenarios has been developed by comparing and combining different point selection methods and applying various metamodelling techniques.
\end{abstract}

Keywords: Hybrid Electric Vehicle (HEV), Electric Vehicle (EV), optimization

\section{Introduction}

The automotive industry is a very competitive industry. In order to gain competitive advantages it is imperative that innovative vehicles are developed rapidly as well as economically. Furthermore, due to global awareness, concern, as well as political- and legislative focus on environmental protection and conservation significantly increases the design and engineering challenges within the automotive industry. In general, vehicle manufacturers focus upon the development of lightweight vehicle structures, which must comply with stringent safety regulations. With reduction of mass as the main objective, it is imperative that the required functionality of the vehicle structure is retained or perhaps even improved. Besides NVH and fatigue life, crash performance is one of the most important attributes to be considered, partially due to the legislative requirements. In order to meet these difficult challenges for lightweight vehicle design and development; the use of emerging computational optimisation techniques is increasing. The first step of the "ideal"optimisation process, with respect to mass reduction, is therefore the use of topology optimisation, providing definitions of load paths within the specified vehicle design envelope, the design volume. This optimisation phase removes "surplus" material, which does not efficiently contribute to the structures ability to react the applied loading. The postprocessing of the topology optimisation results is a very important stage of the overall process. This is because the topology optimisation model is not likely to have incorporated all potential variations of crash-scenarios. Hence sensitivity studies of the topology are required in order to 
further ensure the structural integrity of the topology optimisation results. The definition, as well as execution and post-processing of the topology optimisation results utilising NCAP equivalent load cases will be the focus of the first part of this paper. The second step of the proposed "ideal" optimisation process consists of generating a metamodel, which is subsequently used to obtain a more refined / detailed vehicle structure, by determining the definitions of local areas, i.e. shape- and size optimisation of cross-sections. The metamodel based optimisation does however facilitate a loss in "model accuracy", as will be apparent from section 3.1. Furthermore, this step is extremely CPU time and resource intensive, relative to the remaining steps of the overall optimisation process. This is primarily due to the large number of test-models required to generate the single metamodel, in addition to the complexity of these test-models. The necessity to capture the highly non-linear behaviour of the crashstructure during certain load cases also adds significant complexity to the definition and use of the metamodel. In order to allow the sizing optimisation to convert towards a robust and safe design, it is important that the hyper response surfaces generated for the metamodel are simultaneously smooth as well as accurate. The second part of this report will document step two presented above, i.e. metamodelling. This will be conducted by means of an investigating into a proposed metamodel optimisation strategy, the objective of which is to minimise the mass of the front crash structure of a Hybrid Electrical Vehicle (HEV), when subjected to a $35 \mathrm{mph}$ front impact scenario. Several researches have investigated the potential for employing shape- and size optimisation for crash structures, such as Marklund [1] Jansson et al. [2], Etman [3], Yang et al. [4] and Schramm and Thomas [5]. However, these investigations utilised individual response surface methodologies only. In contrast, Forsberg and Nilsson [6] examined and compared various metamodelling techniques implemented within LS-OPT [7], and their potential suitability for crash structure optimisation. This paper will investigate these various sampling methods, and draw conclusions on the most appropriate metamodel for minimising the mass of an HEV front crash structure subject to NCAP based loading scenarios.

\section{Topology Optimisation}

The initial part of the paper presents how the structural load paths were extracted from an initial design volume, i.e. CAD model, by employing $\mathrm{FE}$ based linear static topology optimisation.

The design volume utilised is illustrated in Fig. 1. The approximate maximum exterior dimensions of the design volume were: $(\mathrm{x}, \mathrm{y}, \mathrm{z}) 3865$ $\mathrm{mm} \times 1850 \mathrm{~mm}$ x $1530 \mathrm{~mm}$.

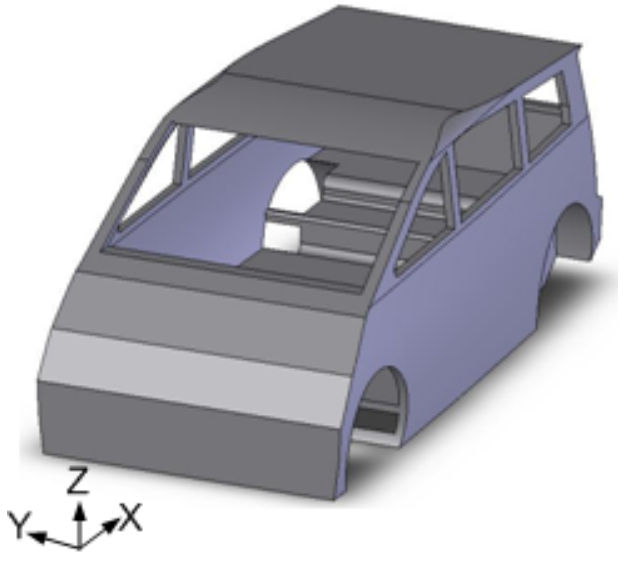

Figure 1: Design Volume

\subsection{Discretisation}

The above illustrated design volume was meshed using first order solid tetra elements, with an average size of $25.0 \mathrm{~mm}$. This led to the generation of approximately 103000 nodes and 527000 elements.

\subsection{Load cases}

The load cases utilised in the optimisation process were intended to be representative of the worst case legislative and NCAP $(35 \mathrm{mph})$ rigid wall dynamic impact loading scenarios. Therefore, a total of six loading scenarios were defined, these are listed below, and have been converted into equivalent static loads based on crash pulse patterns of vehicles with equivalent mass (less than $1200 \mathrm{~kg}$ ) [8]:

1. Front impact, Offset Deformable Barrier (ODB).

2. Pole impact.

3. Side barrier impact.

4. Roof crush: A-pillar.

5. Low speed centred rear impact.

6. High speed rear impact.

The approximate locations of the above defined loading scenarios are illustrated in Fig. 2. 

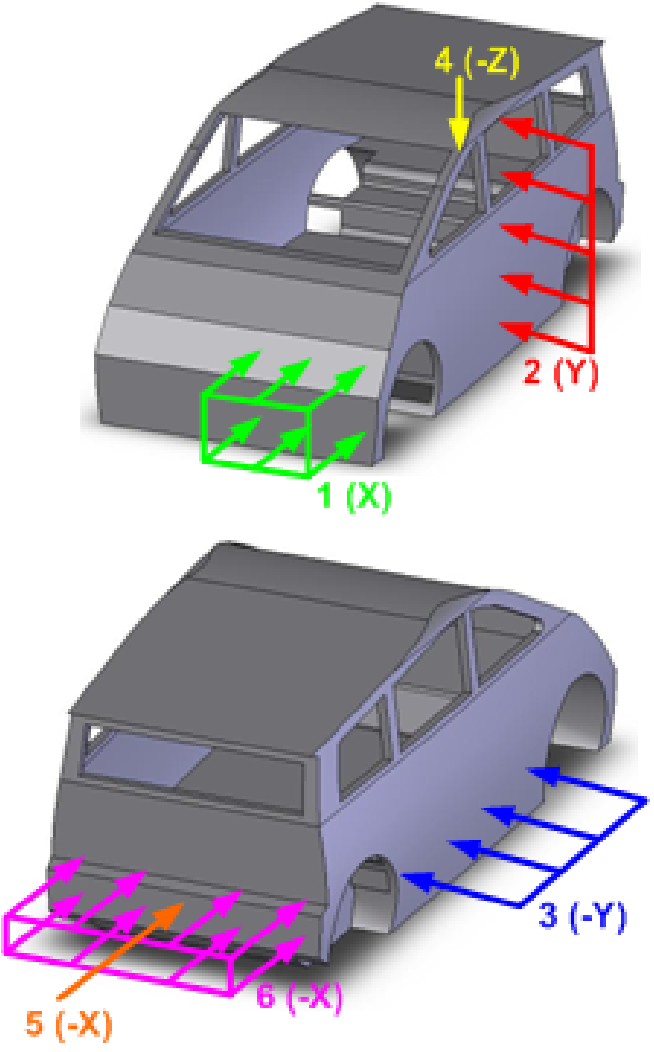

Figure 2: Loading scenarios
The employed linear static topology optimisation algorithms were based on the Solid Isotropic Material with Penalisation (SIMP) interpolation scheme [11], stipulating that the relationship between the stiffness matrix $[k]$ and the volumetric mass density $(\rho)$ was defined by the "power law for representation of elasticity properties", equation 1, [12]:

$$
\underline{[k]}(\rho)=\rho^{p}[k]
$$

In equation $1,[k]$ is the penalised stiffness matrix, and $\mathrm{p}$ is the penalisation factor, which is used to determine the "type" of relationship between $[k]$ and $\rho$. As long as $\mathrm{p}$ is equal to 1.0 the two are directly proportional, as illustrated in Fig. 3.

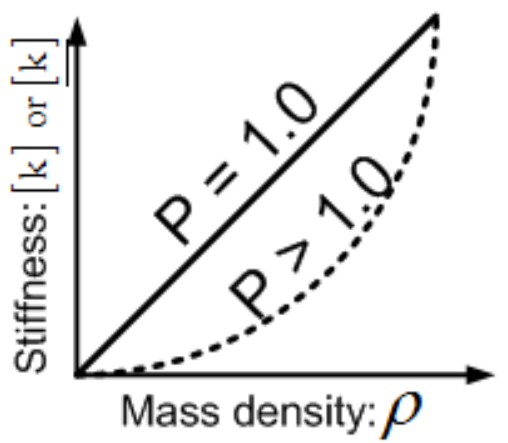

Figure 3: Relationship between $[k]$ and $\rho$

\subsection{Material}

The material model used for the topology optimisation is linear elastic, utilising the material characteristics of a standard grade steel:

- Young's Modulus, E: $210 \mathrm{GPa}$.

- Poisson's ratio: $\nu$ : 0.3.

- Volumetric mass density; $\rho: 7850 \mathrm{~kg} / \mathrm{m}^{3}$

\subsection{Material Law}

The current state of the art topology optimisation methods are based upon an implicit linear solving algorithm which is very well suited for structural stiffness design. These methods can however not predict any non-linearity, let alone complex buckling events, such as the collapse of a front longitudinal member [9]. Within these limitations, it has previously been documented that the solution provided by this linear solver provided a "reasonable" topology for the safety cell, roof and floor [10].
This relationship can be adjusted, by varying $\mathrm{p}$ with the effects as indicated in Fig. 3. The reason for adjusting this relationship is typically to penalise intermediate density values, in order to avoid "vague" definitions of topology, this is also sometimes referred to as "checkerboard effect". However, initial analyses revealed that this was not a widespread problem for the models in question. Therefore in the remainder of this paper the value of $p$ will be 1.0 , i.e. a linear relationship between the stiffness matrix $[k]$ and the mass density $\rho$ will exist.

\subsection{Boundary Conditions}

The boundary condition used for the topology optimisation models was Inertia Relief (IR). The following information relating to IR is primarily based upon Barnett \& Widrick [13]. Inertia relief utilises a significantly different approach to obtain load equilibrium of an FE model. In this approach no Degree Of Freedom (DOF) of any nodes are constrained, due to Boundary Conditions (BC), as is the case with the "traditional" Single Point Constraints (SPC). Instead, inertia relief works by balancing the external loading with inertial loads and accelerations within the structure itself. This is specifically done by "adding" an extra displacement-dependent load 
to the load vector: $\{F\}$ in equation 2 .

$$
\{F\}=[k] \cdot\{u\}
$$

In equation $2,[k]$ represents the stiffness matrix, and $\{u\}$ represents the unknown nodal displacements. The additional terms of the stiffness matrix, due to IR can be appreciated by observing equation 3 .

$$
\{F\}=\left[k_{I R}\right] \cdot\{u\}=\left[\begin{array}{cc}
{[k]} & 0 \\
0 & {\left[k_{A D D}\right]}
\end{array}\right] \cdot\{u\}
$$

In equation $3\left[k_{I R}\right]$ is the stiffness matrix of the IR model, $[k]$ is the "original" stiffness matrix, i.e. the one listed in equation 2 , and $\left[k_{A D D}\right]$ represents the additional terms in the stiffness matrix, caused by the usage of IR. Thus by comparing equation 2 to equation 3 , the fundamental difference between an SPC model and an IR model can be appreciated. With the description of the general model setup complete, the following chapter will present the outcomes of the topology optimisation study.

\subsection{Topology Optimisation Results}

Following the choice of material laws and (IR) boundary condition strategy, a resulting vehicle BIW topology optimisation was obtained, this is illustrated in Fig. 4.

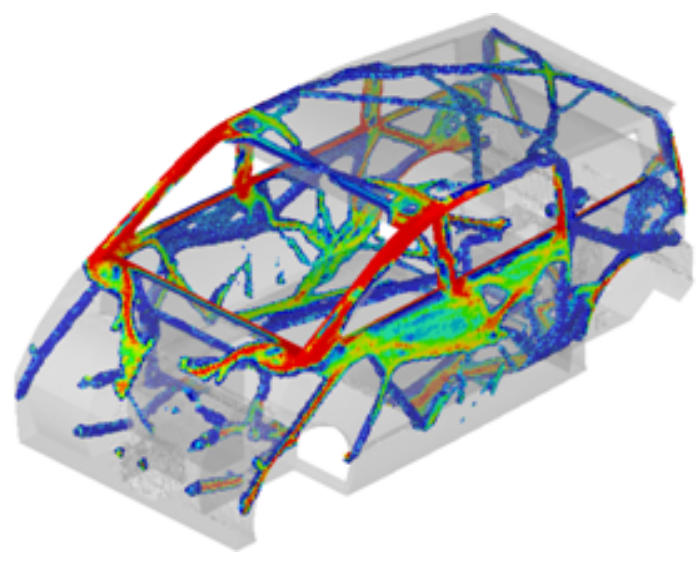

Figure 4: Topology optimisation results

As previously explained, the post-processing of the topology optimisation steps is imperative, especially with respect to variations of load cases, therefore a series of sensitivity studies were conducted in this connection.

The topology optimisation study also included investigation of the optimal centre of mass (CM) locations with respect to key HEV components such as battery packs, feasibility and practicality of the CM locations, as well as vehicle dynamics. Further information on the topology optimisation study can be found in [10], [14] and [15]. The proposed vehicle structure in Fig. 4, is "unconventional" when compared to most modern day vehicles. This was particularly evident for the roof area, where the "traditional" roofbows were replaced by a more triangular lattice structure. This was most likely caused by the usage of a linear static solver. The safety cell does however meet deformation targets [10] and recommends bracings in the doors area, as indicated in Fig. 4.

\subsection{Front Structure Development}

The development of the front structure could not be developed using linear static topology optimisation, due to the large and non-linear deformations anticipated in the crumple zone during crash scenarios. It was however concluded that the remaining topology optimisation results (including the safety cell) provide a good "starting point", i.e. "BIW draft", primarily because large deformations, including non-linear behaviour is not anticipated at these locations. For the development of the front structure, current state of the art crash structure designs were investigated [8]. The outcome of this investigation was used to specify performance requirements, relating to e.g. pulse and intrusion. These were subsequently used to define optimisation constraints for the development of the front crash structure. The lightweight vehicle front crash structure which served as the starting point for the development of the metamodel is illustrated in Fig. 5.

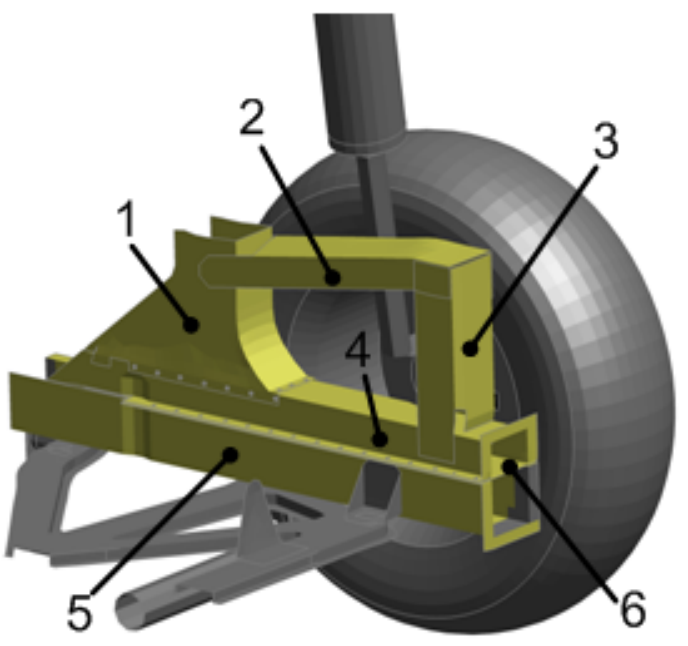

Figure 5: Illustration of initial crash structure

The crash structure illustrated in Figure 5 was originally engineered by Ravenhall [16]. It consists of six main parts:

1. Shotgun 1

2. Shotgun 2

3. Shotgun 3

4. Longitudinal top section 


\section{Longitudinal lower section}

\section{Longitudinal inner reinforcement}

In essence, the structure utilises the bending mode of the longitudinal beams to absorb the impact energy, thus creating the desired pulse profile [16]. The initial mass of the front crash structure illustrated in Fig. 5 is $31.3 \mathrm{~kg}$. During a 35 mph front crash NCAP scenario the maximum intrusion was determined to be $542.5 \mathrm{~mm}$, with a maximum crash pulse magnitude of $42.9 \cdot \mathrm{g}$ occurring at approximately $60 \mathrm{~ms}$. Fig. 6 illustrates the deformed crash structure at $25 \mathrm{~ms}$.

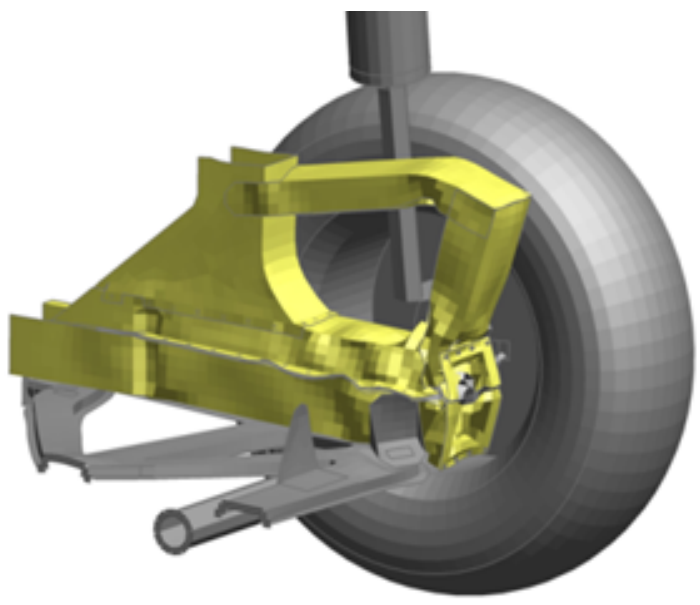

Figure 6: Crash structure at $25 \mathrm{~ms}$ exposed to a 35 mph front crash scenario

The intrusion and pulse magnitudes as functions of time during the $35 \mathrm{mph}$ front crash scenario are visualised in Fig. 7. This particular model utilised a total vehicle mass of $1200 \mathrm{~kg}$. The graph of the pulse illustrated in Fig. 7 was been smoothed using a C60 filter and is of "standard shape" [8].

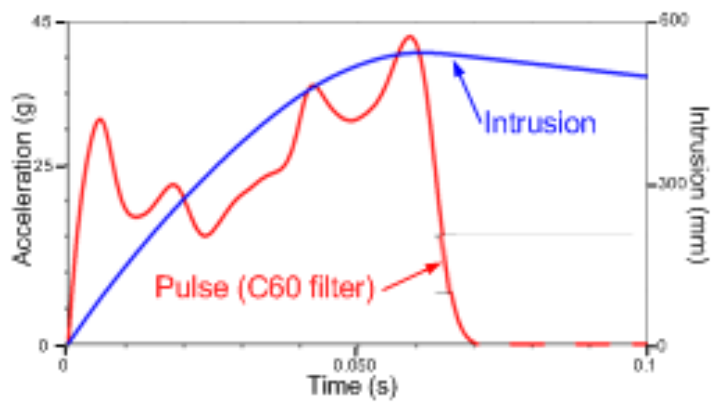

Figure 7: Intrusion and pulse as functions of time, for a $35 \mathrm{mph}$ front crash scenario using the initial front end structure

The pulse profile in Fig. 7 had an initial lower pulse "plateau" with an averaged pulse of approximately $20 . \mathrm{g}$ in the time interval between 0 and $40 \mathrm{~ms}$. The first pulse peak occurring at 5 ms was caused by the initial stress wave propagation throughout the crash structure. This originated from the initial contact with the crash barrier, and should therefore not be taken into account. The higher pulse "plateau" occurring between 40 and $60 \mathrm{~ms}$ had a maximum pulse of $42 \cdot \mathrm{g}$. These higher g values were caused by the front end structure "locking up" towards the end of the crash scenario, this is caused by the limited length of the primary front end crash structure.

\section{Shape- and size Optimisation}

This part of the study was intended to evaluate the potential for developing BIW crash structures based upon the outcome of topology optimisation. The intention was to utilise shape- and size optimisations to determine the "ideal" optimisation process with respect to crash scenarios.

Because of the large number of parameters associated with such a study it was not feasible to fully consider all potential permutations, therefore a simplified mathematical model was required, a metamodel. A metamodel works by means of a multi-dimensional response surface which is used to "estimate" the response of the full model. In order to successfully create a response surface it is imperative to assess the influence of the individual design variables against the objective, which in this case was to minimise the mass. For the purpose of creating this surface a series of "key experimental models" were needed. The approach for selecting these was to utilise Design of Experiments (DOE), which can be implemented in a variety of different ways. The potential for implementing individual sampling methodologies to obtain the above will be discussed in the subsequent sections. Before this was conducted it was necessary to define the actual design variables. This led to a series of complex challenges; one of which was parameterisation. This was essential in order to enable the optimisation of local areas of the crash structure, with respect to the individual design variables associated with the metamodel. Consequently, a vast number of computational permutations were required in order to complete the parameterisation. This meant that a very large number of nonlinear FE crash models had to be solved, which in turn led to substantial CPU costs, as solving a single model using 12 CPUs took approximately 1 hour. Another important challenge was therefore to balance the required CPU time with the accuracy of the metamodel.

\subsection{Design Variables}

A total of six design variables were strategically chosen, including two shape variables, all of which are illustrated in Fig. 8. The design variables were selected based on their estimated potential for significantly affecting the overall crashworthiness of the front end structure. Appropriate constraints were subsequently applied to the individual design variables, such as mini- 
mum (and maximum) thickness values, individual distances etc.

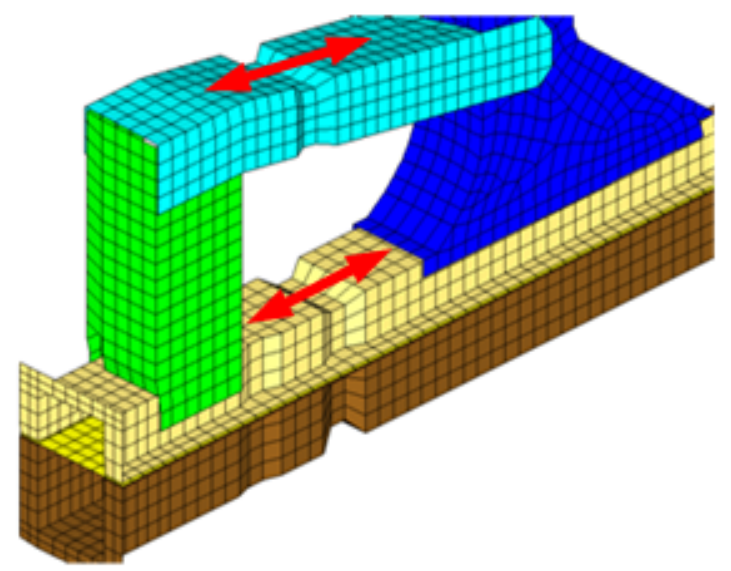

Figure 8: Design variables

With the design variables specified the DOE studies could commence.The following sections will discuss the potential for employing various sampling methods for DOE, with respect to creating the aforementioned metamodel for shapeand size optimisation in relation to crashworthiness and minimising mass.

\subsection{Full Factorial versus Hammersley}

The first DOE study involved the creation of 144 design points using firstly Full Factorial Sampling (FFS) and secondly Hammersley Sampling (HS). All response surfaces were created for the two shape variables illustrated in Fig. 8, which in essence was the locations of the "recesses" along "shotgun 2" (V1) and the "longitudinal" (V2) respectively, Fig. 5, as well as the intrusion response. Initially Least Square Regression (LSR) approximation for both sampling methods were used to create response surfaces. Not only did the LSR create linear response surfaces, due to the first order approximation, but it also created a fundamental difference between the respective response surfaces, as they had different angles in the design space, as illustrated in Fig. 9.

As indicated in Fig. 9, the first order LSR created significantly different metamodels for the individual sampling methods, thus an accurate model could not be created or identified using this approach.

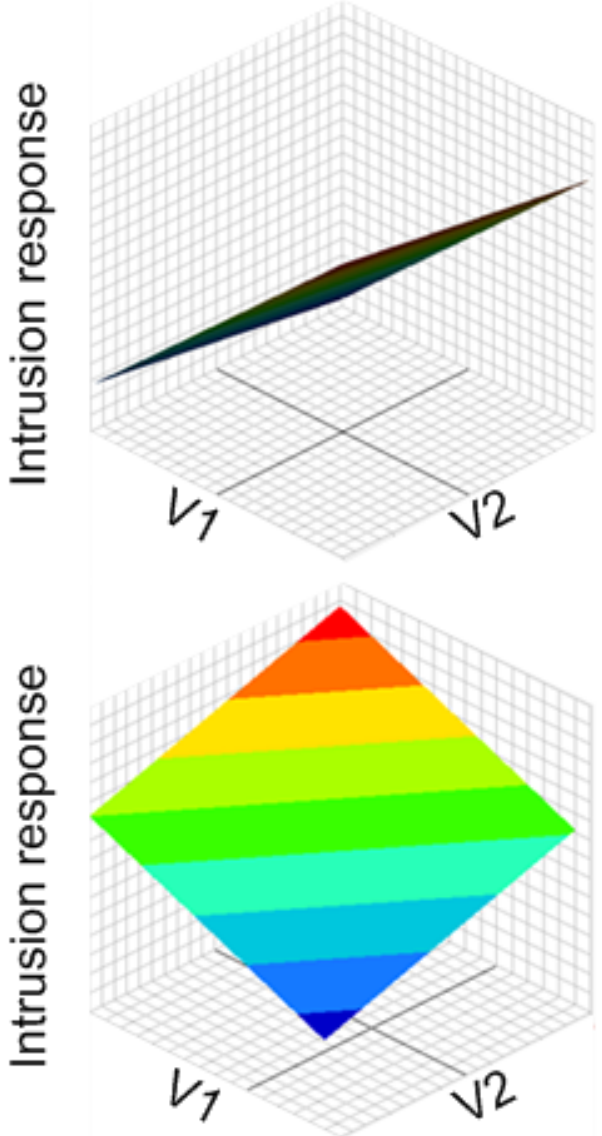

Figure 9: LSR response surfaces using HS (upper) and FFS (lower)

Subsequently Moving Least Squares (MLS) approximation for both sampling methods was used to create a new set of response surfaces. These substantiated that the HS based approximation created accurate response surfaces, and that the response surfaces based upon FFS was rendered insufficient, as shall be further discussed in section 3.4 .

Finally response surfaces were created using the Kriging interpolation method. As with the MLS approach, the response surface based on FFS combined with Kriging interpolation was deemed to be inadequate. It was found that kriging interpolation method combined with HS created a suitable response surface. However, the results also indicated that issues regarding the robustness for the subsequent optimisation would persist, as the response range was very wide, and instable runs would be included in the metamodel.

\subsection{Central Composite versus Hammer- sley Sampling}

For the second DOE investigation, the rectangular Central Composite Sampling (CCS) was compared to HS, this study consisted of 77 design points. The first step of this DOE study once 
again consisted of creating LSR response surfaces. As with the first study documented in section 3.2 these response surfaces were found to be "insufficiently shaped" for conducting accurate optimisation. As a result, a substantial number of sampling points would be required to ensure that the resulting metamodel was of sufficient accuracy.

Secondly, two response surfaces using MLS were created. Both were deemed to have "appropriate" shapes, as was previously found to be the case for MLS, HS using 144 design points. The response surfaces created using 77 design points were however found to have "insufficient" definition in the vicinity of the actual design points. The use of the Kriging interpolation method in this context was found to lead to non-smooth response surfaces, particularly in connection with HS. This was found to be especially evident at $\mathrm{V} 1=1$ and $\mathrm{V} 2=1$, as illustrated in Fig. 10, and would thereby lead to a non-robust optimisation model.

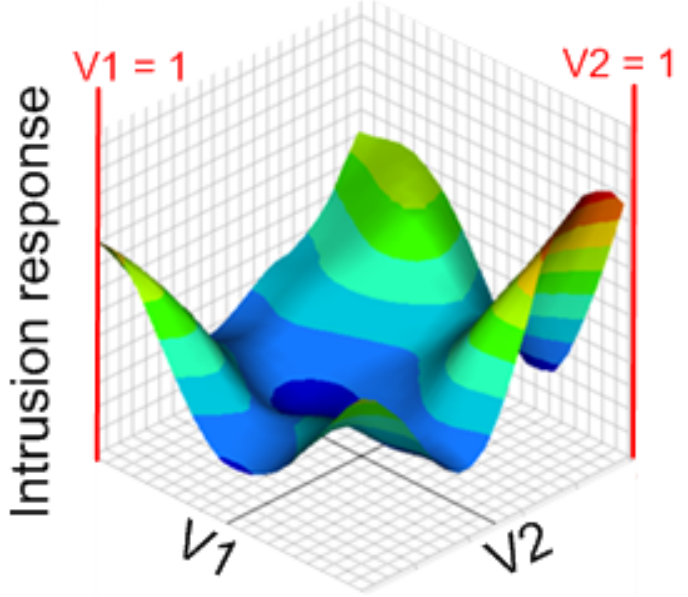

Figure 10: Kriging response surface using HS, with 77 design points

\subsection{Combining FFS and HS}

Based on the above findings, an additional three response surfaces were created. These were based on the combined findings of the FFS and the HS 144 design point samplings presented in section 3.2 , and utilised $4^{\text {th }}$ order LSR, $3^{\text {rd }}$ order MLS as well as the Kriging interpolation method. The rationale for this coupling approach was to investigate the potential for "stabilising" the response surfaces at the extreme points, a problem previously highlighted by Fig. 10. The coupling approach in combination with the above interpolation methods should ensure a "reasonable" level of accuracy at the boundaries of the response surface, whilst simultaneously maintaining the accuracy within the design space. The three response surfaces created in this context are illustrated in Fig. 11.

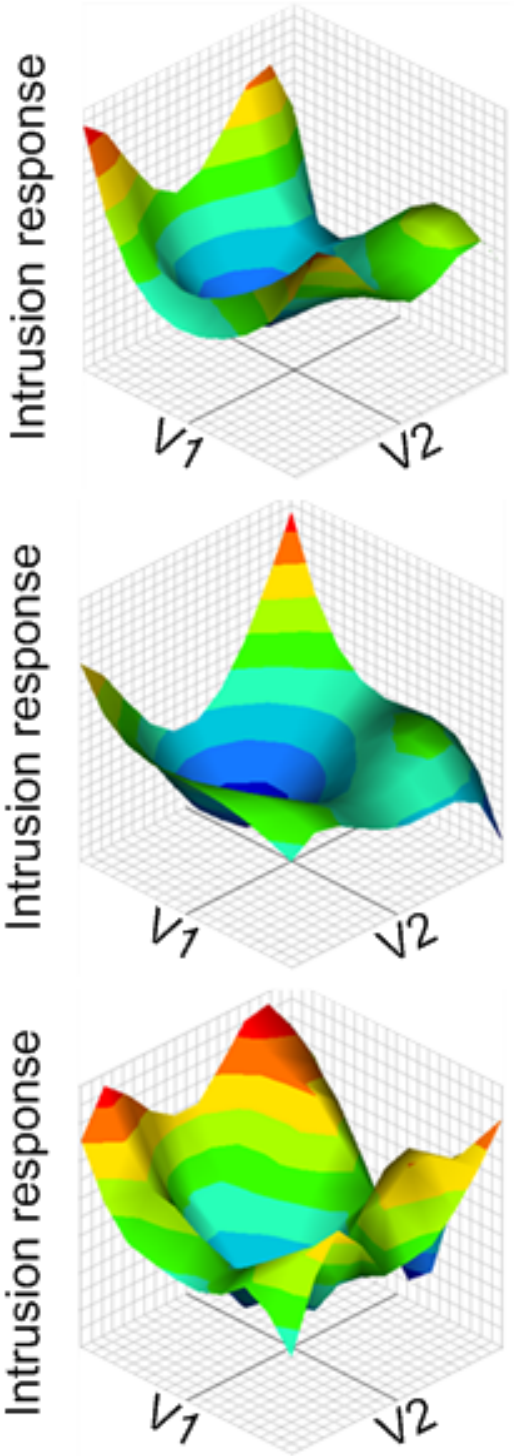

Figure 11: Response surfaces based on combined FFS and HS (144 runs), using $4^{\text {th }}$ order LSR (top), $3^{\text {rd }}$ order MLS (middle) and Kriging (bottom)

As illustrated in Fig. 11, all three response surfaces have "similar" shapes, and adhere to the response surface initially generated from the MLS metamodel using HS (144 runs). The response surface in Fig. 11 created using Kriging had sharp edges and steep slopes. These characteristics of a response surface led to a non-robust optimisation, and could thereby be disregarded. The error resulting from using the LSR and MLS metamodels of Fig. 11 are presented in Fig. 12, as a function of the individual design points. 


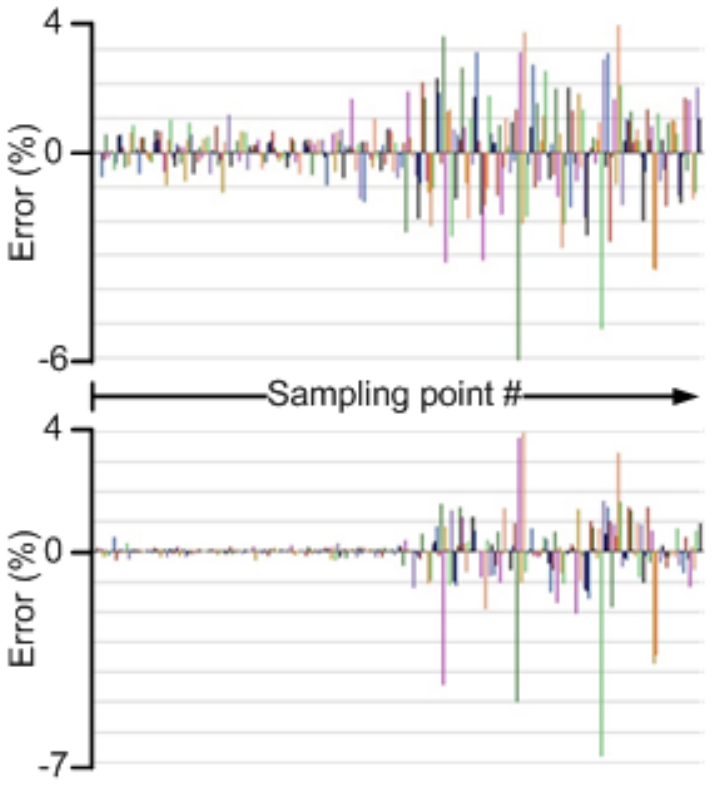

Figure 12: Percentage error of $4^{\text {th }}$ order LSR (upper) and $3^{r d}$ order MLS (lower)

Based on the results represented by Fig. 11 and Fig. 12 it became evident that the MLS approximation had the capability to form a smooth and simultaneously accurate response surface. In addition, the MLS model had the ability to ignore unstable runs, this does however result in a higher percentage error of those specific design points, clearly visible in Fig. 12.

The higher order LSR also generated an "adequate" metamodel; however, when compared to the MLS model the latter was found to "perform better". In addition, the CPU time required to create the MLS response surface was only a fraction of the CPU time required to create the LSR equivalent.

\subsection{Combination of FFS CCS and HS}

Fig. 13 presents equivalent response surfaces to those illustrated in Fig. 11; the difference being that Fig. 13 includes the contribution for the rectangular CCS, consisting of 77 design points, initially presented in Fig. 10.

The response surfaces in Fig. 13 created using $4^{\text {th }}$ order LSR and $3^{\text {rd }}$ order MLS were almost "identical" to the ones presented in Fig. 11. The two Kriging surfaces evidently differed drastically, as sharp slopes emerged at the edges of the surface in Fig. 13.
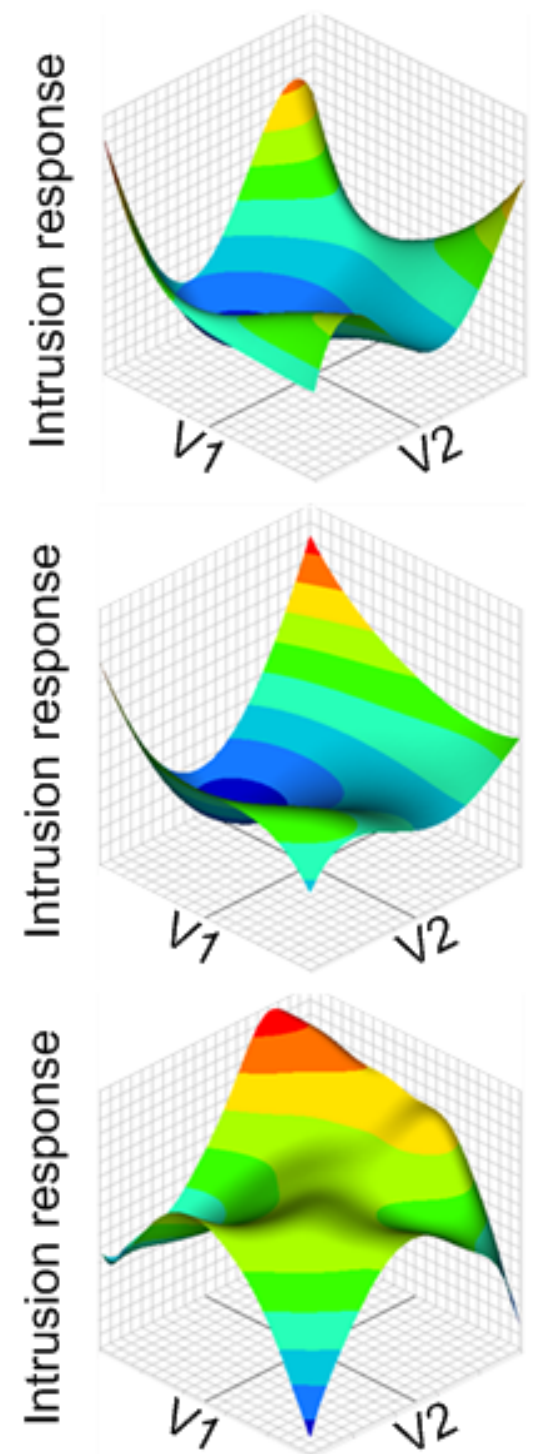

Figure 13: Response surfaces based on combined FFS (144 runs), HS (144 runs) and CCS (77 runs); using $4^{\text {th }}$ order LSR (top), $3^{r d}$ order MLS (middle) and Kriging (bottom)

In order to determine the accuracy and indeed the suitability of the metamodels for accurate crashworthiness optimisation, it was necessary to more carefully consider the effects of introducing the CCS results. Fig. 14 represent the "crosssectional" views of the three response surfaces in Fig. 13, with the shape variable V1 set to zero.

The pink curve in Fig. 14 represents the metamodel created using MLS. As Fig. 14 reveals, this curve did not change significantly by the addition of the CCS results. Hence, the "interior" points as well as the response surface boundaries were already "adequately" determined, prior to the addition of the CCS results, which did therefore not improve the metamodel.

Before the CCS points were taken into account both the LSR and the Kriging curves individually 

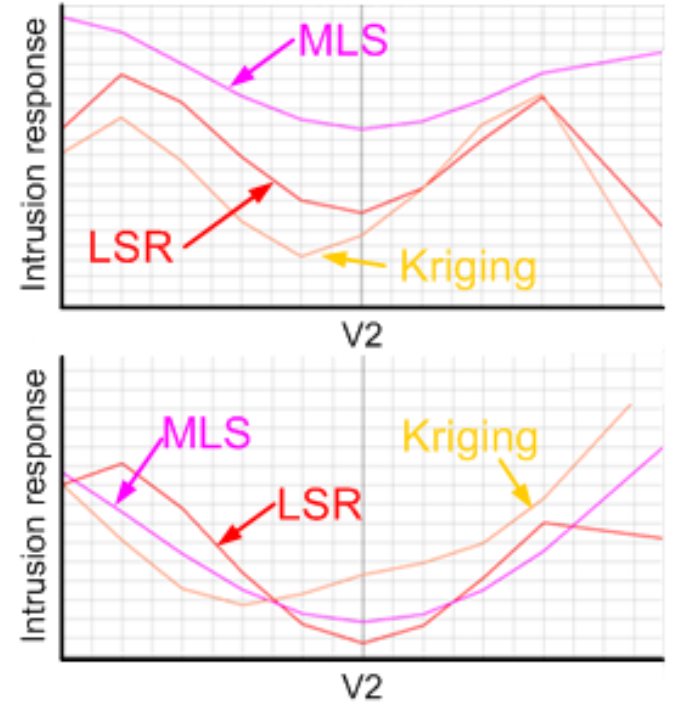

Figure 14: Cross-sectional view of the combined FFS and HS (upper) and with added CCS (lower)

deviated significantly with respect to the metamodel created by MLS, as illustrated in the upper part of Fig. 14. When the CCS results, which mainly defined the design points within the design space, where subsequently considered, the metamodel generated by MLS became similar to the other two. The metamodels created by LSR and Kriging moved towards the metamodel produced by the MLS. Consequently, the MLS approximation created "correct" metamodels with fewer design points as compared to the LSR and Kriging approximations.

\subsection{Variable Screening}

As previously mentioned variable screening is crucial for the identification and elimination of "unnecessary" variables within multi variable problems; thus decreasing the amount of design points necessary to represent the actual system. In most cases regression coefficients are used to determine dispensable variables. The sampling methodologies and associated response surfaces discussed above were based on the two shape variables V1 and V2 illustrated in Fig. 8. However, Fig. 8 also contain the remaining 4 design variables V3 to V6. Fig. 15 represents the main influences on the individual 6 variables upon the intrusion response using the FFS (144 runs) and a Plackett-Burman (36 runs) design. The PlackettBurman can be interpreted as a "scaled down" version of the FFS run, it is a so-called fractional factorial design [12]. Fig. 15 was obtained using ANalysis Of Variance (ANOVA) [12].
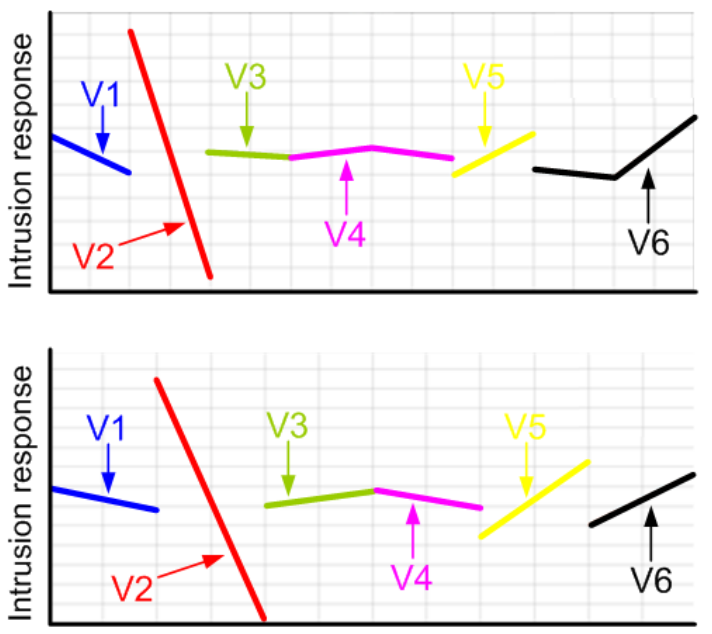

Figure 15: Individual design variable influence upon intrusion response; FFS model (144 runs)(upper) and Plackett-Burman(36 runs) (lower)

Fig. 15 thus provides an ANOVA; a graphic representation of the influence of each individual design variable upon the intrusion response. As Fig. 15 reveals, the effects identified by use of the FFS design were very similar to those identified by the Plackett-Burman design. The PlackettBurman did however have the obvious advantage that the results were obtained by 36 runs as opposed to 144 , resulting in a significant decrease in CPU costs.

\section{Optimised Design}

Based on the findings and results presented above, it was concluded that the most appropriate methodology for creating an accurate and smooth metamodel for robust optimisation, with respect to crashwothiness and mass reduction, was the moving least squares method combined with HS. On this basis a combined shape- and size optimisation of the crash structure illustrated in Fig. 5 , and the design variables illustrated in Fig. 8 was conducted. The objective of which was, as previously mentioned, to minimise the mass of the structure; the load case consisted of a $35 \mathrm{mph}$ front crash scenario. The resulting optimised design of the front crash structure is illustrated in Fig. 16. 


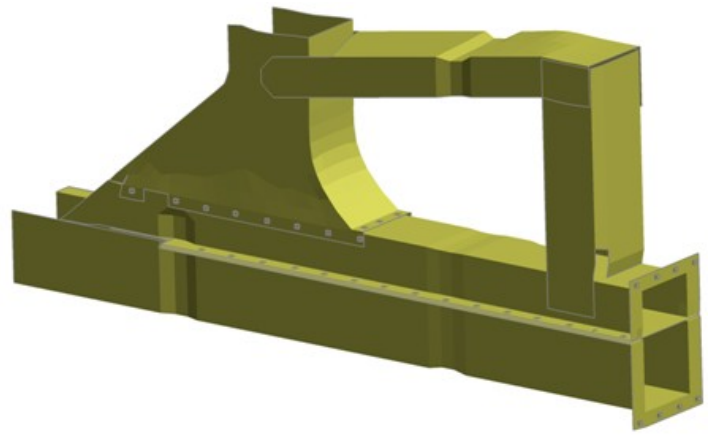

Figure 16: Optimised crash structure

When comparing Fig. 16 to Fig. 5 it was difficult to notice any major differences. This was not unexpected, as the original structure of Fig. 5 was already well engineered, and "fit for purpose". Nevertheless, the optimised structure of Fig. 16 represented a mass reduction of $0,91 \mathrm{~kg}$ or approximately $3 \%$ relative to the original values. The pulse profile and intrusion are shown in Fig. 17, which also contains the original profiles, previously presented in Fig. 7.

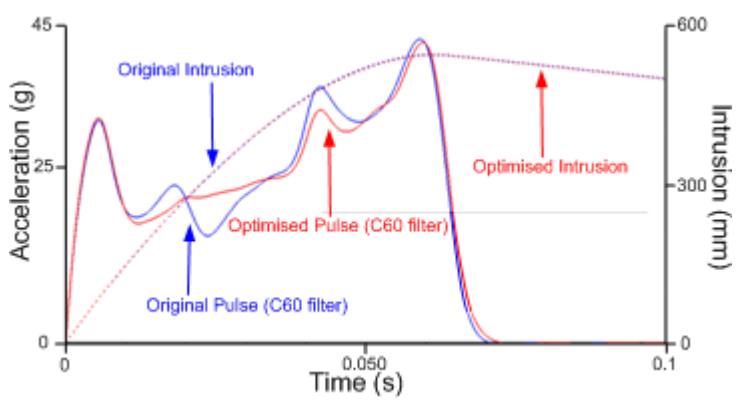

Figure 17: Intrusion and pulse as functions of time, for original and optimised crash structures

The two pulse profiles illustrated in Fig. 7 did not display large differences; however, the pulse peak value of the optimised model was slightly lower than that of the original. The intrusion responses from both models remained unchanged, with identical maximum values.

All of the above results show that the shape- and size optimisation was successful, as the objective of reducing the mass was met, in addition the "crashworthiness" constraints of pulse and intrusion values were not violated, in fact the maximum pulse magnitude was slightly reduced. Although the mass was only reduced by a modest $3 \%$, which may not be of huge importance considering the overall vehicle mass, the study has justified that the shape- and optimisation approach worked, and provided results as intended, which is of significant importance with respect to developing structural optimisation algorithms in general, and more importantly their potential for application to crashworthiness.

\section{Conclusion and Next Steps}

This paper has highlighted some of the key findings of a study into the potential for applying structural optimisation algorithms within the field of crashworthiness, given the objective of minimising mass. The paper commenced with a study on HEV BIW architecture design using topology optimisation, followed by shape- and size optimisation for developing the detailed geometry of the front crash structure. It was found that topology optimisation on its own was adequate for safety cage development, but not for the automatic generation of the front end crash structure. The linear static topology optimisation can be used for BIW development, however the engineering interpretation of the results are essential. To "automatically" develop crash structures from design envelopes, thus minimising the "manual design work", a topology optimisation algorithm using non-linear FEA (currently not available in commercial software) could provide an improved "starting point" for the shape- and size optimisation. If this was to be achieved, the "originally engineered" structure in Fig. 5 could theoretically be replaced with the direct outcome of topology optimisation. Research into the development of such an algorithm is presently ongoing. The second part of the study demonstrated that shape- and size optimisation of crash structure components can indeed be successfully completed. However, this process is very complex, due to various factors such as initial design, design space, sampling method, metamodelling technique, optimisation algorithm, mesh and morphing strategy, which all need to be respected.

The initial design was found to be a significant factor which ultimately determined the success of the shape- and size optimisation. As mentioned above, a non-linear topology optimisation algorithm could "automatically" deliver an improved proposal for crumple zones, thus enhancing the combined efficiency of the entire optimisation process, which potentially could significantly decrease the time used for the overall design process.

The shape- and size optimisation study concluded that the most appropriate methodology for creating an accurate and smooth metamodel for robust optimisation with respect to crashwothiness and mass reduction, was the MLS method combined with HS.

This method can be used to efficiently and accurately optimise the cross sectional properties (i.e. shape and size) of a BIW crash structure, whilst meeting NCAP requirements. This was substantiated by dynamic (explicit) FE modeling verifying the outcomes of the shape- and size topology optimisation.

\section{Acknowledgements}

The authors of this paper would like to thank colleagues from Coventry University, Tata Motors European Technology Centre (TMETC), 
Jaguar Land Rover (JLR), Warwick Manufacturing Group (WMG) and other contributors to the Low Carbon Vehicle Technology Project (LCVTP) for supplying data leading to the optimisation studies presented in this paper.

\section{References}

[1] Marklund P. O., Nilsson L. Optimization of a car body component subjected to impact, Structural Multidisciplinary Optimization, 21:383-92, 2001.

[2] Jansson T, Redhe M, Nilsson L. Using surrogate models and response surfaces in structural optimization - with application to crashworthiness design and sheet metal forming, Structural Multi-disciplinary Optimization, 25:129-40,2003.

[3] Etman LFP. Optimization of multibody systems using approximations concepts, Technical University Eindhoven, Eindhoven, 1997.

[4] Yang R-J, Gu L, Tho CH, SobieszczanskiSobieski J. Multidisciplinary design optimization of a full vehicle with high performance computing AIAA Paper No. 2001-1273, 2001.

[5] Schramm U, Thomas H. Crashworthiness design using structural optimization, AIAA Paper No. 98-4729, 1998.

[6] Forsberg J, Nilsson L. Evaluation of response surface methodologies used in crashworthiness optimization, International Journal of Impact Engineering, 32 (2006) 759-777.

[7] Livermore Software Technology Corporation. LS-OPT manual, 2011.

[8] NHTSA, http://www.nhtsa.gov/Research/Databases+and+Software, accessed on 2011-12-01.

[9] Christensen J, Bastien C, Blundell M V Effects of roof crush loading scenario upon body in white using topology optimisation, International Journal of Crashworthiness, DOI:10.1080/13588265.2011.625640, 2011.

[10] Bastien C, Christensen J. Towards the Light weighting of Low Carbon Vehicle Architectures using Topology Optimisation, EHTC , Bonn, Germany, 2011.

[11] M. P. Bendse, O. Sigmund. Topology Optimization - Theory Methods and Applications, Berlin, Springer Verlag, 2003.
[12] Altair Engineering, Inc. Altair Hyperworks manual, Troy, 2010.

[13] R. Barnett and T. W. Widrick. ClosedForm Static Analysis With Inertia Relief and Displacement-Dependent Loads Using a MSC/NASTRAN DMAP Alter, http://ntrs.nasa.gov/archive/nasa /casi.ntrs.nasa.gov/19950013233_1995113233.pdf, accessed 2011-03-01

[14] Bastien C, Christensen J, Dickison M, Nicholson R, Gittens A, Tomlin O. Topology Optimisation of a Body in White for Low Carbon Vehicle Technology Project, EHTC, Versailles, France, 2010.

[15] Christensen J, Bastien C, Blundell M V, Gittens A, Tomlin O. Lightweight Hybrid Electrical Vehicle Structural Topology Optimisation Investigation Focusing on Crashworthiness, International Journal of Vehicle Structures and Systems,ISSN 0975-3540, Volume 3, Issue 2, 2011.

[16] Ravenhall N. Crash pulse analysis of current vehicles and concept design analysis, Coventry University.

\section{Authors}

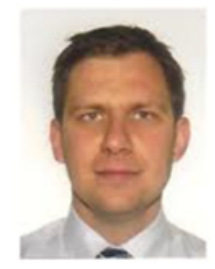

Christophe Bastien MSc, BEng (Hons), CEng, MIMechE, is a principal lecturer and the MSc Automotive Engineering Programme Manager at Coventry University. He has over 15 years of industrial and academic experience with FEA and crashworthiness. $\mathrm{He}$ is currently leading the research into the lightweighting of electrical vehicle architectures. He has filed 19 patents in the field of vehicle and highway engineering safety, and is currently undertaking a $\mathrm{PhD}$ in occupant biomechanics.

Jesper Christensen MSc, BSc, CEng, MIMechE, is a Lecturer in Stress Analysis at Coventry University. The past 2 years of his career has been spent focusing on structural optimisation in connection with crashworthiness, primarily in relation to HEV vehicles, for which he has published several recent papers. He is currently undertaking a $\mathrm{PhD}$ within the field of structural optimisation. 

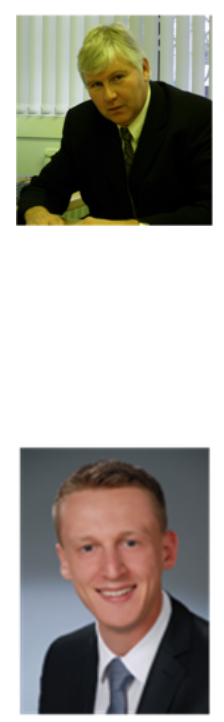

Professor Michael Blundell, $\mathrm{PhD}, \mathrm{MSc}, \mathrm{BSc}$ (Hons), FIMechE, CEng. Mike has over 30 years of experience in academia and also in industry with companies and institutions such as Boeing and the Ministry of Defence. Continuous experience throughout his career on the development and application of CAE and Virtual Engineering in the marine, nuclear, automotive and aerospace industries.He has published more than 95 papers, has authored an engineering textbook and is a regular reviewer of government funded research proposals, reports and publications.

Jakovs Kurakins Msc, Bsc, is a recent graduate from Coventry University. His individual masters project entitled: "Investigation of Optimisation Strategies for Multi Variable Problems with focus on Lightweight Vehicle Front Structure Development for LCVTP" was supervised by Christophe Bastien and Jesper Christensen. 